\title{
Benchmarking of stope design and reconciliation practices
}

\author{
Y Potvin Australian Centre for Geomechanics, Australia \\ KR Woodward Australian Centre for Geomechanics, Australia \\ B McFadyen Université Laval, Canada \\ I Thin KSCA Geomechanics Pty Ltd, Australia \\ D Grant BHP, Australia
}

\begin{abstract}
In 2019, the Australian Centre for Geomechanics organised a benchmarking workshop in Perth, Australia, followed by a second workshop in Rouyn-Noranda, Canada, under the sponsorship of BHP Olympic Dam. The workshops aimed to document the current stope design and reconciliation practices in open stoping operations from Australia and Canada. A total of 19 underground mines-including copper, gold, and base metal mines-provided data to the benchmarking study. The benchmarking study has provided data to further our understanding of the stope design and reconciliation process.
\end{abstract}

The process can be simplified into four main steps:

1. Life of Mine stope planning.

2. Stope design.

3. Operation and execution.

4. Reconciliation.

The main input and output for each of the steps, which are common to all mines, will be summarised in the paper. Standard and advanced practices within each step of the process will be described. Our interpretation of what are the main gaps in the design and reconciliation process will be discussed.

Keywords: stope design, stope reconciliation, benchmarking

\section{Introduction}

The Australian Centre for Geomechanics conducted a benchmarking study on stope design and reconciliation practices in underground mines. The first workshop was held in Perth, Australia, on 12 June 2019 and a second workshop was held in Rouyn-Noranda, Canada, on 5 August 2019.

The purpose of the workshops was to understand and document the current stope design and reconciliation practices at mine sites. A total of 19 underground mines-including copper, gold and base metal mines-provided data to the benchmarking study. This paper documents and discusses the outcomes of this workshop.

\section{General information and some key observations}

All mines that participated in the benchmarking study used a variation of the longhole open stoping methods, except for one mine which implemented inclined room and pillar mining. From the 18 mines using open stope mining, two are strictly narrow vein mining, six are open stoping in a massive orebody, and 10 have a medium orebody width varying from 5-15 m using a mix of longitudinal and relatively small transverse open stopes.

From the 19 mines, 13 mines use paste fill; three mines use cemented rock fill or rock fill, and three mines (including the room and pillar mine) use no fill at all. 
It was found that the stope design and reconciliation approaches and, more specifically, the focus adopted at mines, were highly influenced by their context (orebody shape and orientation, grade distribution, geotechnical and geological settings, etc.). The following are some observations derived from the discussions held during the workshop and do not necessarily reflect the authors' opinion:

- All mines reconcile volumes, tonnes, and grade for each stope.

- This feeds into an overall periodic reconciliation of tonnes and grade for each mine. This process is driven by management and generally led by the geology department.

- Mines using small stopes and rapid turnaround are less likely to perform thorough reconciliation process (beyond the points above) on individual stopes.

- Mines with medium to large stopes use a more intricate design and reconciliation process. This is facilitated by longer lead times and more reliance on individual stope performance.

- Narrow vein mines are very focussed on hanging wall overbreak and equivalent linear ore loss (ELOS). This is because a small amount of overbreak translates into large percentage dilution.

- Lower grade mines with 'hard' ore/waste boundaries are very focussed on overbreak in general. This is because the profit margin of individual stopes can be small and sensitive to dilution.

- At least one mine with a massive orebody and disseminated grade in the waste was tolerant of overbreak because the economic impact of overbreak was relatively lower.

- Not many mines record overbreak and underbreak on an individual stope surface basis.

- At most mines, one or more major factors are attributed to be the root cause of overbreak, but this is rarely backed-up with quantitative data.

- Not many mines focus on underbreak from individual stope surfaces, however, at least two high grade mines reported monitoring underbreak from the footwall.

\section{The stope design process}

The idealised design and reconciliation timeline from Olympic Dam-a massive orebody with very large open stopes-is shown in Figure 1. For smaller mines, the process follows similar steps but the timeline is compressed significantly. Typically, the mine site stope design (third step) is undertaken approximately three months prior to stope extraction. For some mines using rapid small turnaround stopes, the last three steps in the timeline are even more compressed and completed within a few weeks. Only one mine from the workshop discussed a formal risk ranking assessment process at the mine site stope planning stage.

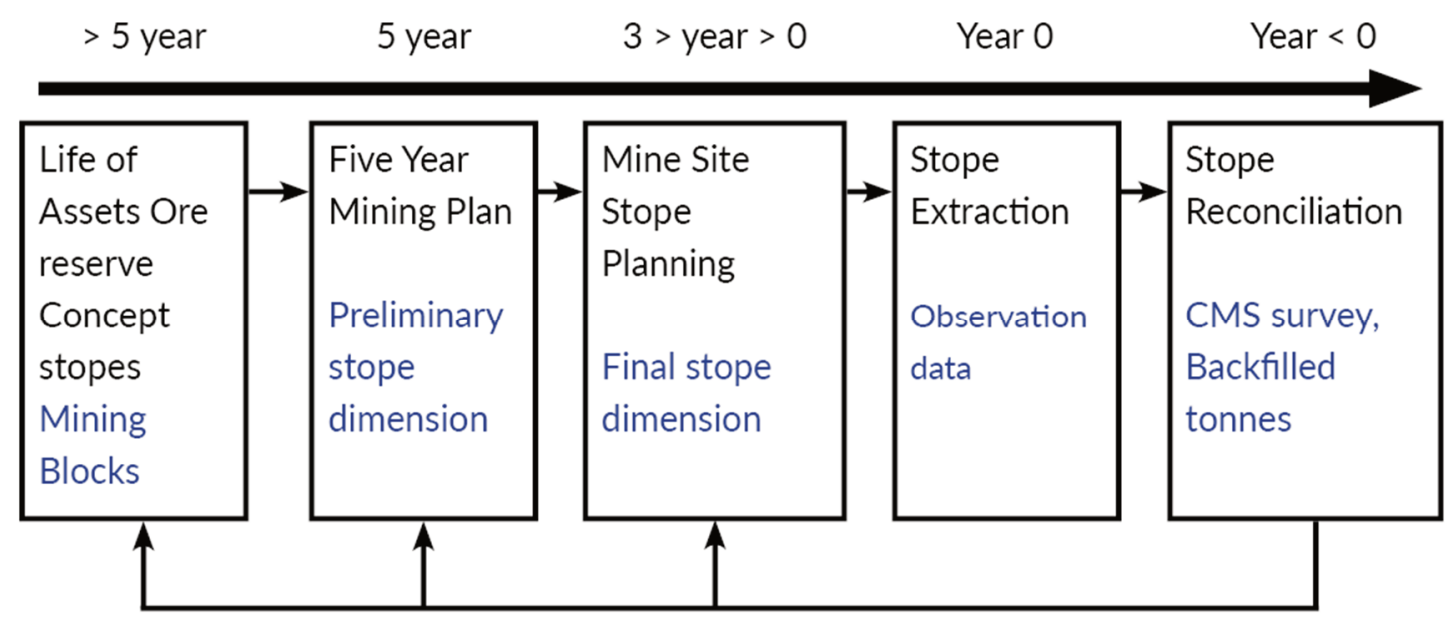

Figure 1 Stope design and reconciliation macro-process at Olympic Dam (Potvin et al. 2012) 


\subsection{Generic stope shape and dimensions}

The starting point of the process is to develop generic stope shapes and dimensions during the life-of-mine (LOM) or major mining blocks planning, which is updated regularly. The responsibility for completion of this step often lies with the long-term planning department.

The standard input for this step is generally a lower confidence geological/block model, an already set stope shape and dimension or, alternatively, rules to generate stope shape and dimension following generic geotechnical guidelines, and a set value for recovery and dilution.

The standard output tends to be a 'cookie-cutter' stope shape and dimensions for the mining block including a preliminary extraction sequence, mining and development plan and economic evaluation. Pre-set overbreak, and underbreak values, are generally applied to every stope. At that stage, the focus is on tonnes, grade, and strategic mining schedule.

For the mines using smaller stopes, the economic and preliminary stope design (second box in the process shown in Figure 1) is generally lumped with the previous LoM step. For the massive orebody/large stopes, the stope planning progresses from a long-term plan to a more immediate mining plan. Adjustments to the generic stope shape and dimension are made at that stage by the planning engineers to produce an individual stope with mineable shapes and dimensions that suits the most up-to-date orebody and mining requirements. The planning extends to mine accesses, preliminary stoping sequence, ventilation, blasting, backfill, services and infrastructure. A preliminary economic assessment is often performed at that stage based on the tonnes and grade derived from the mineable stope shape. Advanced practices for this step were not identified.

\subsection{Stope design}

Essentially, the preliminary stope designs from the previous step are refined through more detailed geology and geotechnical investigations before being passed on to the drill and blast design stage. The output is a stope design note containing all information and backup engineering, decisions, and specifications on how to mine the stope. Some of the key output items include:

- Design stope shape based on cut-off grade, tonnes and operating physicals.

- Stope wireframe.

- Ground support requirements.

- Slot opening, ring design and charging.

- Fill specification and delivery requirements.

- Ventilation implication.

- Predicted overbreak.

- Seismic protocols.

- Barricading and other actions taken in response to stope specific risks.

Backfill design is initiated at that stage but will only be completed after stope extraction is completed to confirm the volumes required. This is not illustrated in the simplified flowcharts of Figure 1.

\subsubsection{Geology investigation}

If required, definition diamond drilling of the area is completed ahead of the stope design work. The orebody delineation is updated. Core logging of the new holes and geological mapping of relevant excavations enable an update to the geotechnical model of the area. A review of the potential influence of major structures and lithology is often performed. 
Although not often available at mine sites, the authors believe that an up-to-date structural model is important. Furthermore, there may be ore recovery at the mill and metallurgical implications from overbroken waste rock.

\subsubsection{Geotechnical investigation}

Underground mapping and rock mass classification of selected development drives also contribute to updating the geotechnical model. Some versions of the stability graphs and ELOS graph or local empirical relationships are applied to verify stope stability, and to produce forecast overbreak (or ELOS) quantities. The need for cable bolt reinforcement of walls and/or stope crown is also assessed.

Many mines rely on numerical modelling at that stage to understand how the stresses change with the extraction of stopes (effect of stress path on the infrastructure) and identify potential stress induced problem areas. The stoping sequence may be revised based on this work. Seismic hazard will be evaluated for mining induced seismicity in seismically active mines.

Some form of a feedback loop is often used for future design, implementing the learnings from previous stopes outcomes in similar conditions. Having empirical charts calibrated with extensive site data is considered good practice.

\subsection{Drill and blast design}

The designed stopes from the previous step are provided to the drill and blast engineer to define the drilling pattern and charging plan that aims to produce the specified shape and designed dimensions. This step is often executed only a few days or less before the drilling commences.

The blast design is ideally informed by the grade contour and the general philosophy favouring high recovery versus low dilution, as these are conflicting goals. High recovery will use aggressive drilling and blasting practices (small or no stand-off distance, even over-drill), while low dilution will have a conservative stand-off distance. This is the only step of the stope design process where underbreak control is considered. Good practice will rely on extensive interaction between the drill and blast engineer and geomechanics personnel to produce the final design.

\section{Operation and execution}

The execution stage of the process can be very comprehensive and complex and is outside of the scope of this paper. The main input is the stope design notes and all the mine plans issued (including drill and blast) to execute the design. Most mines have a comprehensive sign-off process involving specialist engineers such as geotechnical, backfill, ventilation, etc., as well as management. The main output regarding a 'signed-off' stope design and reconciliation process will be the stope void wireframe, operational data (for example, tonnes hauled), grade sampling results, and any geotechnical observation/monitoring data.

\section{Stope reconciliation}

The stope reconciliation stage is initiated with the Cavity Monitoring System (CMS) survey. CMS surveys are sometimes carried out repeatedly during the stope production after individual blasts. In other instances, there are only one or two surveys taken once the stope is empty. The survey is performed when the stope is empty and is used to define the required volume to be filled and as an input for preparing the 'Fill Note'. Therefore, the last stage of stope design, which is finalising the fill requirements for an individual stope, occurs in parallel with the mining operation and execution stage and after the reconciliation process is initiated.

Stope reconciliation has different meanings to different people. This is because the reconciliation can be done from a production point of view, a geotechnical point of view, a geological point of view, a drill and blast point of view, and a filling point of view. 
The overall process of stope reconciliation is not always owned by any one department of the mine. The geology department, however, has an unavoidable role to play in monitoring tonnes and grades from individual stopes and periodically reconcile these with the mill output.

\subsection{Cavity Monitoring System survey}

All participating mines used the CMS (Figure 2a) to survey the resultant stope void, either during the production progress after individual blasts/during mucking or when the final void was complete. To minimise shadowing, many mines perform surveys from the top and bottom of the stope when access is available (or accessible sublevels when dealing with a multi-lift stope). Other mines complement the CMS survey with a borehole laser (CALS) (Figure 2b). There is also an increasing interest in the use of drones to perform stope void surveys. Many of the participating mines used a resolution of $1^{\circ} \times 1^{\circ}$. High accuracy surveys can assist in identifying adverse structures, causing overbreak.

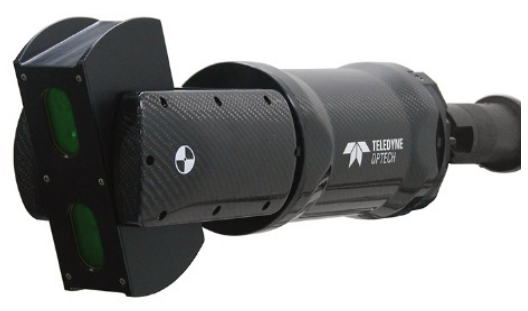

(a)

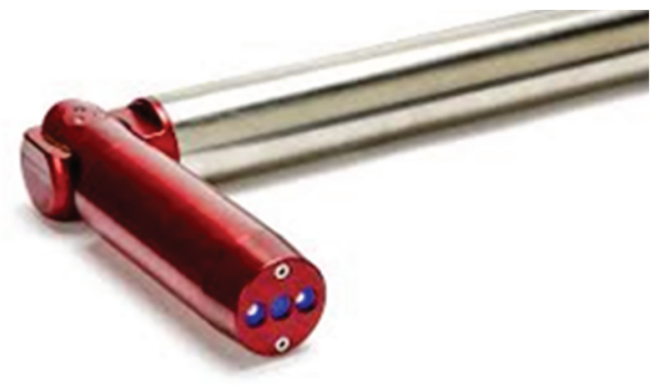

(b)

Figure 2 (a) The Optech CMS V500 (CR Kennedy Survey Solutions 2020); (b) The CALS borehole laser (Austech Surveying and Mapping 2020)

One major issue reported during the benchmarking is the requirement to use multiple types of software to complete the reconciliation process. This is not only time-consuming and cumbersome, but it also limits the capability of conducting in-depth analyses of the root cause or causes of overbreak and underbreak.

Commonly, most mines use the raw survey data (a 3D point cloud often from multiple scans) and CMS software to produce a triangulated mesh (Figure 3).

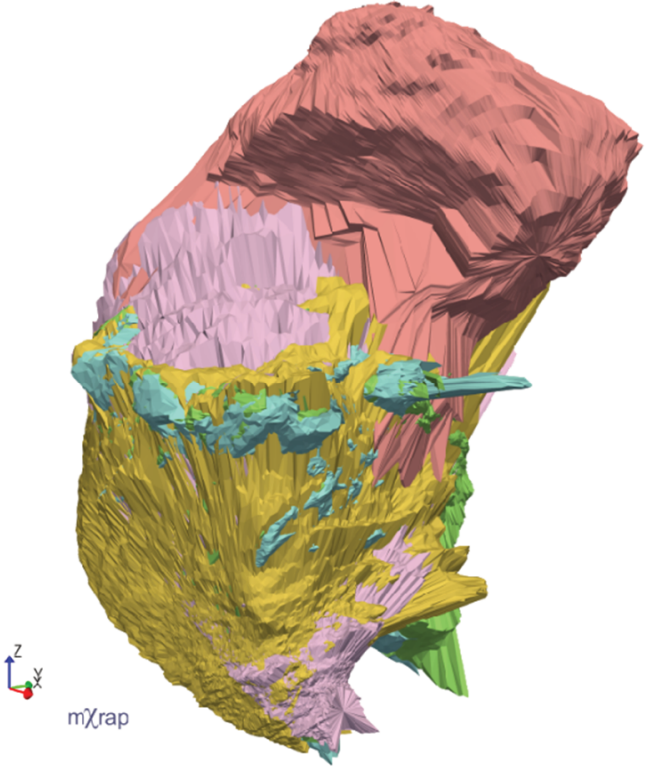

(a)

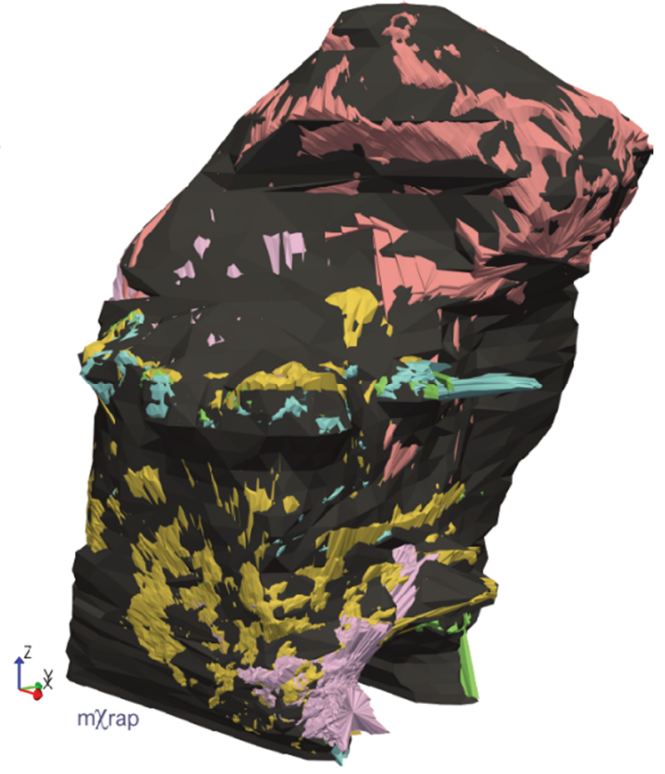

(b)

Figure 3 Example of Cavity Monitoring System survey: (a) Point cloud and (b) Triangulated mesh 
The CMS mesh is exported into other software which is then compared with a mesh of the planned stope shape (or drill and blast shape) to calculate overbreak and underbreak. The data manipulation often involves slicing the mesh and overlaying different data from different software.

A wide variety of software is used at the different mines for these analyses, including but not limited to:

- Deswik (the most used software).

- Datamine.

- Surpac.

- Minesight.

- Leapfrog.

- Vulcan.

- In-house software.

- OneNote.

- Excel.

- Autocad/Promine.

- Gem 4D.

- Rhino 6.

- Carlson CMS.

Up to recently, there was no integrated platform to perform stope reconciliation, and specific software is required for the various reconciliation tasks. Geotechnical data, blasting data, survey data and geological model data are generally accessible through different software. All assist in understanding the root cause of overbreak and underbreak and as such, are used for different reconciliation tasks.

There are several differences between reconciliation practices at the different benchmarking mine sites. For example, some mines compare the CMS survey to the generic stope shape (assuming it is the same as the drilled and blast shape), while other mines generate a new design mesh based on the drill and blast design. The latter involves more data manipulation but provides a more realistic representation of the planned stope, hence a more accurate estimation of overbreak and underbreak.

Some mines also prefer to exclude the volume of existing development drives because these tonnes have already been accounted for during the development mining phase. In narrow vein mining, for example, stope development can be a substantial proportion of the production. One issue with excluding the development mining drives is that they may contribute significantly to overbreak by undercutting walls (footwall or hanging wall), or to underbreak due to poor positioning of the development, sometimes under geological control. Therefore, at least their position should be accessible for the purpose of reconciliation and identifying root causes of overbreak and underbreak.

The above differences in practices underline the difficulties in standardising stope reconciliation processes.

\subsection{Backfill planning}

As previously mentioned, the backfill requirements are based on results from the final CMS survey from which the shape and volume of the void are estimated. The Fill Note is then prepared, which completes the stope design step of the process. At least one mine mentioned doing a 'stope void review', where backfill placed tonnes/volume and predicted placed tonnes/volume are compared.

When the stope is filled, this completes the stope execution and production step of the process. The stope reconciliation was initiated in parallel to these activities and continues until the feedback loop is completed. 


\subsection{Estimation of overbreak}

All mines that have taken part in the benchmarking systematically estimate for all stopes overbreak by volume and tonnes, which is also often expressed as percentage dilution. They then reconcile the tonnes and metal recovery for the individual stopes and periodically for all stopes mined during the period, against the mill recovery. This can be challenging given the complexity of the ore handeling (and re-handling) at some mines, as well as the ore stockpiled on the surface.

From the 19 participating mines:

- Nine mines use ELOS, which implies that overbreak is estimated at least for one individual wall. This is typically the hanging wall, in addition to reconciling for the whole stope.

- 11 mines calculate overbreak and/or ELOS for all individual stope walls to enable more in-depth analysis of overbreak.

- 16 mines are using backfill and 12 mines specifically separate backfill overbreak from the solid rock mass overbreak. This could be considered standard practice for mines using fill.

\subsection{Estimation of underbreak}

Underbreak can have a significant impact on the economic performance of a stope. The amount of underbreak is largely a function of the stope shape and the effectiveness of drill and blast design and, more specifically, drilling pattern and stand-off distance. It is also acknowledged that there is a human factor involved in drill and blast that can impact on the resultant underbreak, but this is difficult to quantify. Similar to overbreak, underbreak is measured in volumes and can be expressed in tonnes. It can be compiled for the whole stope or for individual stope surfaces.

From the benchmarking study, 14 out of 19 mines are measuring underbreak for reconciliation purpose, and 12 of them are assigning a grade to the underbreak to assess metal recovery.

Apart perhaps from an informal review of CMS from the drill and blast engineers, only two mines systematically compile underbreak per stope surface to look at the root cause or to establish the correlation between blasting factors and underbreak. Compiling underbreak for individual stope surfaces is considered advanced practice.

\subsection{Root cause of overbreak and underbreak}

While few mines perform a root cause analysis of their underbreak, all mines have a good appreciation of the main causes of overbreak. Amongst the common factors affecting overbreak mentioned, the most common are:

- Faults or shear zones.

- Weak lithology.

- Drives notching (undercutting) the wall.

- Adverse stope geometry.

- Drilling and blasting.

- Time the stope is left open.

- Cable bolt performance.

- Stress and seismic response.

- All aspects of QA/QC. 
When a stope does not perform as predicted, generally some more in-depth investigations are conducted. Otherwise, overbreak is quantified without formal root cause analysis. The response to performance deviation is very much focussed on fixing overbreak and lower than estimated grade. Higher than expected grade and underbreak do not receive the same attention.

\section{Feedback loop}

The last step of the overall stope design and reconciliation process is to 'close the loop' by using as many learnings as possible achieved during the reconciliation process, in future designs. We believe that this is how value can be created from the stope design and reconciliation process.

\subsection{Use of historical data for dilution forecasting and informing ore reserves}

Every participating mine in the benchmark study has used the historical overbreak data, in some way or another, to forecast dilution in future stope designs. This is standard practice. Most of the mines have developed their own graphical design tools based on published empirical methods.

Although some mines would rely on historical underbreak data to inform their recovery factors, none of the mines have an empirical (graphical or other) method to forecast underbreak.

The forecasting of overbreak and nominal values of underbreak is used by 11 out of 19 mines to inform the ore reserves declaration.

\subsection{Backfill performance}

Although no formal methods to calibrate fill design was shown during the workshop, 13 mines reported using the reconciliation data to assess the performance of fill mass stability. It is believed that the data is only used qualitatively to assess fill performance.

\subsection{Drill and blast performance}

Most mines distribute the CMS survey to the drill and blast engineers for reconciliation purposes. However, no data or methods were presented to confirm that this reconciliation with drill and blast goes beyond qualitative comparison. Without $Q A / Q C$ measurements, the identification of the root cause of the drill and blast malfunction is generally not possible.

\section{Summary}

The starting point of the study was the generic process illustrated in Figure 1 . The benchmarking study has provided data to further our understanding of the stope design and reconciliation process. Firstly, it is proposed that the process can be further simplified into four main steps:

1. LoM stope planning.

2. Stope design.

3. Operation and execution.

4. Reconciliation.

Secondly, the main input and output for each of the steps, which are common to all participating mines, have been identified. These are illustrated in the updated flowchart in Figure 4. 


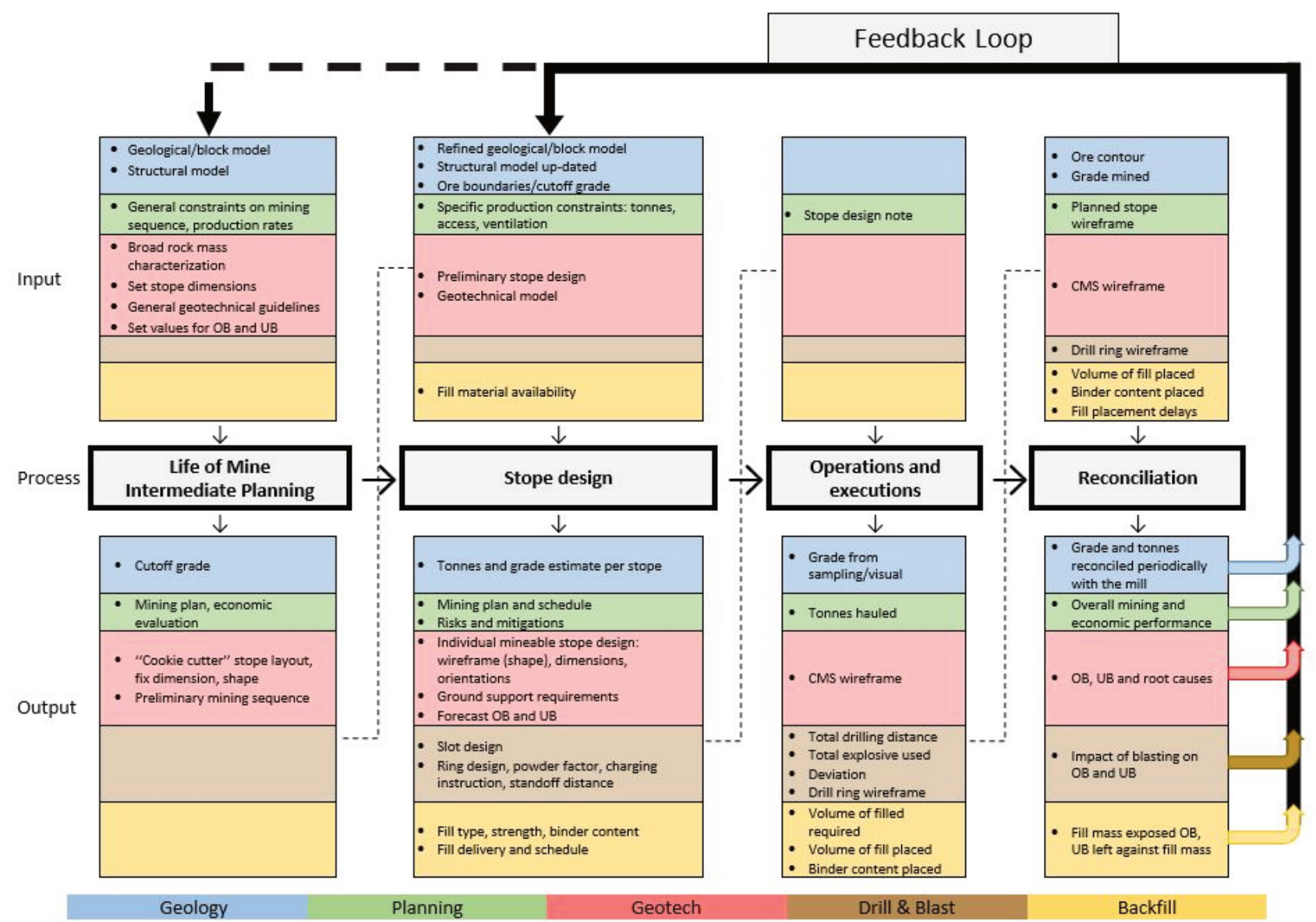

Figure 4 Updated stope and reconciliation flowchart based on the benchmarking

\section{Conclusion}

Ackhoff (1989) described the hierarchy of human mind processes with four distinct levels: at the bottom there is data, which feeds into information, which can then be transformed into knowledge. At the top of the hierarchy comes wisdom. Our interpretation of the status of the stope design and reconciliation process in the industry based on this benchmarking study is:

1. The industry collects lots of data related to stope design, extraction, and reconciliation.

2. For design, these include, amongst other data, extensive core logging and mapping databases compiled into comprehensive geological and geotechnical models. For reconciliation, stope design, void surveys, drill and blast drawings, operational data, etc., are collected.

3. The industry does not have efficient means of transforming the data into usable information.

4. Data manipulation is time-consuming, and mines have insufficient human resources and tools. The reconciliation is made via many different software ( 13 were identified in the benchmarking). None of the software has efficient tools to perform root cause analysis for overbreak and underbreak. As a result, most mines limit their analyses to whole stope (overbreak and underbreak) instead of analysing individual stope surfaces in details.

5. The industry is not transforming information into knowledge.

6. There is no method for forecasting underbreak. The industry still uses generic empirical charts from the 1980s to 1990s to assess the stability of stopes. Blasting and major faults are not included as design parameters with the current methods used but can severely affect overbreak and underbreak outcomes. 
The ultimate goal is wisdom, where good business and safety decisions are underpinning every step of a data-information-knowledge-driven stope design and reconciliation process.

\section{Acknowledgement}

This research would not be possible without industry participation in the workshops and we gratefully acknowledge both the corporate and individual contributions. The authors specifically thank BHP for their sponsorship of these benchmarking workshops and permission to use their data in this paper.

\section{References}

Ackhoff, RL 1989, 'From data to wisdom', Journal of Applied Systems Analysis, vol. 16, pp. 3-9.

Austech Surveying and Mapping 2020, CALS borehole laser, https://www.austechsurvey.com.au/c-als

CR Kennedy Survey Solutions 2020, Optech CMS V500, https://survey.crkennedy.com.au/products/530146-v500/optech-cms-v500system

Potvin, Y, Grant, D \& Mungur, G, 2012, 'Towards a practical stope reconciliation process at Olympic Dam', Proceedings of the Sixth International Conference and Exhibition on Mass Mining (MassMin2012), Canadian Institute of Mining, Metallurgy and Petroleum, Montreal. 